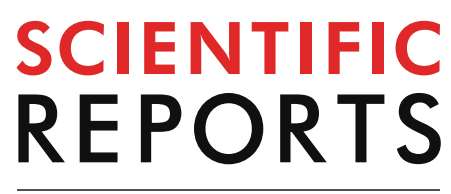

natureresearch

Check for updates

\title{
Plasma sphingosine 1-phosphate concentrations and cardiovascular autonomic neuropathy in individuals with type 2 diabetes
}

\author{
Min Young Chung ${ }^{1}$, Seon-Young Park ${ }^{2}$, Jin Ook Chung ${ }^{1 凶}$, Dong Hyeok Cho ${ }^{1} \&$ \\ Dong Jin Chung ${ }^{1}$
}

The aim of this study was to test the hypothesis that plasma sphingosine 1-phosphate (S1P) levels are associated with the risk of cardiovascular autonomic neuropathy (CAN) in type 2 diabetes patients. This cross-sectional study included 287 individuals with type 2 diabetes. CAN was evaluated using cardiovascular reflex tests. Logistic regression analyses were conducted to assess the relationship between plasma S1P levels and CAN. Plasma S1P concentrations were significantly lower in individuals with CAN than in those without CAN. There was a significant interaction between plasma S1P levels and sex with respect to CAN ( $p$ for interaction $=0.003$ ). When stratified by sex, the association between plasma S1P levels and CAN exhibited a sex difference; in multivariable analysis, plasma S1P levels were significantly associated with CAN in women (odds ratio per standard deviation increase in the log-transformed value, $0.40 ; 95 \%$ confidence interval, $0.23-0.70, p=0.001$ ). However, there was no significant association between plasma S1P and CAN in men. Plasma S1P concentrations were inversely associated with CAN only in women with type 2 diabetes.

Cardiovascular autonomic neuropathy $(\mathrm{CAN})$ is a common but overlooked complication of diabetes mellitus $(\mathrm{DM})^{1}$. CAN involves damage to the autonomic nervous system that innervate the heart and blood vessels ${ }^{2}$, and it is thought to be implicated in silent myocardial ischemia, hemodynamic instability, stroke, cardiovascular mortality, and perioperative cardiovascular liability in individuals with type $2 \mathrm{DM}^{2,3}$. While poor glycemic control, diabetes duration, and hypertension play important roles in the pathogenesis of $\mathrm{CAN}^{4}$, CAN risk cannot be entirely explained by these conventional risk factors, suggesting that other factors may also have an influence on its pathogenesis.

Sphingosine 1-phosphate (S1P), a metabolite of sphingolipids, is a bioactive lipid mediator which regulates cell differentiation, proliferation, apoptosis, and inflammation ${ }^{5}$. Accumulating evidence indicates that S1P plays important physiologic roles in the central and peripheral nervous systems ${ }^{6,7}$. Furthermore, S1P plays a crucial role in neural development ${ }^{6}$. In addition, several investigations have shown that decreased S1P levels may be related to neurodegenerative disease, suggesting that it also plays a neuroprotective role ${ }^{8}$. However, although $\mathrm{S} 1 \mathrm{P}$ has been implicated in neurological disorders, the relationship between S1P and CAN in individuals with type $2 \mathrm{DM}$ has not been clarified.

In the present study, we tested the hypothesis that plasma S1P levels are associated with CAN risk in individuals with type $2 \mathrm{DM}$.

\section{Methods}

Study population. This cross-sectional study included 287 individuals with type $2 \mathrm{DM}$. We consecutively enrolled individuals with type $2 \mathrm{DM}$ who visited the diabetes clinic at our hospital. Type $2 \mathrm{DM}$ was diagnosed based on the "Report of the Expert Committee on the Diagnosis and Classification of Diabetes Mellitus". Individuals were regarded as having hypertension if they had a blood pressure $\geq 140 / 90 \mathrm{mmHg}$ or took anti-

${ }^{1}$ Division of Endocrinology and Metabolism, Department of Internal Medicine, Chonnam National University Medical School, 8 Hak-Dong, Dong-Gu, Gwangju 501-757, Republic of Korea. '2Division of Gastroenterology and Hepatology, Department of Internal Medicine, Chonnam National University Medical School, 8 Hak-Dong, Dong-Gu, Gwangju 501-757, Republic of Korea. ${ }^{\circledR}$ email: imagine-jjo@hanmail.net 
hypertensive drugs. Hyperlipidemia was considered as total cholesterol level $\geq 6.5 \mathrm{mmol} / \mathrm{L}$ and/or triglyceride level $\geq 2.3 \mathrm{mmol} / \mathrm{L}$, or the use of lipid-lowering drugs. We gathered demographic information on smoking status, diabetes duration, and other health factors using standardized inquiries. For women, menopausal status was considered according to self-reported cessation of menses for $\geq 12$ consecutive months not due to pregnancy or medical treatment. The exclusion criteria of this study included a history of glucocorticoid use, advanced renal dysfunction (serum creatinine levels $\geq 176 \mu \mathrm{mol} / \mathrm{l}$ ), liver cirrhosis, pancreatitis, thyrotoxicosis, hypothyroidism, arrhythmia, heart failure, respiratory distress, alcoholism, infection, or malignancy. The study was approved by the ethics committee of Chonnam National University Hospital, and informed consent was obtained from all participants. The study was performed according to the Helsinki Declaration-based ethical principles for medical research involving human subjects.

Methods. After fasting overnight, venous blood samples were collected from the participants. Glycated $\mathrm{Hb}$ (A1C) was analyzed by ion exchange liquid chromatography using an HLC-723-GHbV analyzer (Tosoh, Tokyo, Japan). Serum total cholesterol, triglyceride, low-density lipoprotein cholesterol (LDL-C), and high-density lipoprotein cholesterol (HDL-C) levels were determined using an AU5400 analyzer (Olympus, Tokyo, Japan). S1P levels were determined using commercially available competitive enzyme-linked immunosorbent assay (ELISA) kits (Echelon Biosciences Inc., Salt Lake City, UT) according to the manufacturer's instructions ${ }^{10}$. The intra- and interassay coefficients of variations were $6.5 \%$ and $8.4 \%$, respectively. Albuminuria was identified on the basis of the urine albumin-to-creatinine ratio (UACR) using random urine samples. Estimated glomerular filtration rate (eGFR) was calculated using the Chronic Kidney Disease Epidemiology Collaboration equation ${ }^{11}$. Nephropathy was defined as a UACR $\geq 300 \mathrm{mg} / \mathrm{gCr}$ or $\mathrm{eGFR}<60 \mathrm{ml} / \mathrm{min} / 1.73 \mathrm{~m}^{2}$. After the patients' pupils were dilated, fundoscopy was performed to evaluate diabetic retinopathy. Cardiovascular autonomic dysfunction was assessed by determining heart rate (HR) responses to postural change, deep breathing, and the Valsalva maneuver ${ }^{1,12}$. To evaluate the HR responses to standing, the ratio of the 15 th $\mathrm{R}-\mathrm{R}$ interval to the 30th R-R interval was calculated; a ratio below 1.00 was considered abnormal ${ }^{1,12}$. We assessed the beat-to-beat variation in HR during paced deep breathing by estimating the ratio of the shortest $\mathrm{R}-\mathrm{R}$ interval during inspiration to the longest $\mathrm{R}-\mathrm{R}$ interval during expiration, and compared this to age-related reference values ${ }^{1,12}$. To evaluate HR responses during the Valsalva maneuver, the subject forcefully exhaled through the mouthpiece of a manometer at $40 \mathrm{~mm} \mathrm{Hg}$ for $15 \mathrm{~s}$, and the ratio of the longest R-R interval to the shortest R-R interval during the test was calculated; a ratio below 1.10 was considered abnormal ${ }^{1,12}$. Based on the criteria proposed by the Toronto Diabetic Neuropathy Expert Group, early and definite involvements of cardiovascular autonomic dysfunction were identified as one abnormal and at least two abnormal HR test results, respectively ${ }^{13}$. In the current study, CAN was defined as definite involvement of cardiovascular autonomic dysfunction ${ }^{13}$. Individuals were advised to avoid caffeinated beverages, smoking, and alcohol for the $12 \mathrm{~h}$ preceding the cardiovascular tests and avoid taking the drugs, including antihistamines, diuretics, and beta-blockers, for two days before the tests. Three patients were excluded as they could not perform one or more of the autonomic function tests. In total, 287 subjects were included in this study.

Statistical analysis. Data are presented as means \pm standard deviations (SDs) or frequencies (percentages), unless otherwise remarked. Categorical variables were analyzed using the chi-squared test, and the Mann-Whitney U test or Student's t-test was used for continuous variables. Data with a skewed distribution were logarithmically transformed before regression analysis. In order to compare mean S1P levels according to the degree of cardiovascular autonomic dysfunction, analysis of covariance was performed after adjusting for confounding factors. Using logistic regression models, multivariable analysis adjusting for identified factors and previously known risk factors was conducted to assess the association between S1P levels and CAN. The use of insulin and oral hypoglycemic agents (OHAs) was coded as a dummy variable. A multivariable model with CAN as the dependent variable was used to investigate interactions between S1P level and other covariates. When a significant interaction was identified between S1P and any of the covariates ( $p$ for interaction $<0.05$ ), stratified analysis was then performed. A significant $p$ value of $<0.013$ was used to correct for multiple comparisons. All statistical analyses were conducted using SPSS version 20.0 (SPSS, Chicago, IL, USA). An a level of 0.05 was used for statistical tests.

\section{Results}

Of the total 287 participants, 149 (51.9\%) subjects were men. The prevalence of CAN was $25.1 \%(n=72)$. The clinical characteristics of individuals with type 2 DM according to the presence of CAN are presented in Table 1. Individuals with CAN had a longer duration of diabetes, were older, and had a higher prevalence of hypertension and nephropathy than those without CAN. Plasma S1P concentrations were significantly lower in individuals with CAN than in those without CAN.

A significant interaction was identified between S1P levels and sex $(p=0.003)$ (Supplementary Table1), indicating that the effect of S1P on CAN may be affected by sex. To further evaluate the interaction between S1P and sex, we conducted stratified analysis according to sex. There was no difference in the prevalence of CAN and plasma S1P levels between women and men with type $2 \mathrm{DM}$ (women $22.5 \%$ vs. men $27.5 \%, p=0.324$; women $9.0 \mu \mathrm{mol} / \mathrm{l}$ vs. men $9.3 \mu \mathrm{mol} / 1, p=0.736$, respectively, Fig. 1 and Supplementary Table 2$)$. When stratified by sex, plasma S1P concentrations were significantly lower in women with CAN than in those without CAN (no CAN $9.6 \mu \mathrm{mol} / \mathrm{l}$ vs. CAN [+] $7.4 \mu \mathrm{mol} / \mathrm{l}, p<0.001)$, but there was no significant difference in plasma S1P levels between men with and without CAN (no CAN $9.4 \mu \mathrm{mol} / \mathrm{l}$ vs. CAN [+] $9.2 \mu \mathrm{mol} / 1, p=0.914$ ) (Fig. 1). Average plasma S1P concentrations according to the degree of cardiovascular autonomic dysfunction in women and men are presented in Table 2. In women, plasma S1P levels significantly differed according to the degree of cardiovascular autonomic dysfunction, after adjusting for age, body mass index (BMI), HDL-C, LDL-C, triglycerides, 


\begin{tabular}{|c|c|c|c|}
\hline & CAN (-) & CAN (+) & $p$ value \\
\hline $\mathrm{n}$ & 215 & 72 & \\
\hline Age (years) & $58.6 \pm 12.6$ & $63.7 \pm 9.6$ & $<0.001$ \\
\hline Men (\%) & $108(50.2)$ & $41(56.9)$ & 0.324 \\
\hline Current smoker, n (\%) & $31(14.4)$ & $14(19.4)$ & 0.310 \\
\hline Hypertension, n (\%) & $110(51.2)$ & $49(68.1)$ & 0.013 \\
\hline Hyperlipidemia, n (\%) & $123(57.2)$ & $39(54.2)$ & 0.652 \\
\hline Diabetes duration (years) & $3.0(0.2-10.0)$ & $10.0(2.0-20.0)$ & $<0.001$ \\
\hline Body mass index $\left(\mathrm{kg} / \mathrm{m}^{2}\right)$ & $25.9 \pm 4.4$ & $25.6 \pm 3.3$ & 0.584 \\
\hline Systolic blood pressure (mmHg) & $135.4 \pm 18.5$ & $136.6 \pm 18.9$ & 0.635 \\
\hline Diastolic blood pressure (mmHg) & $78.8 \pm 12.6$ & $75.8 \pm 12.2$ & 0.106 \\
\hline $\mathrm{A} 1 \mathrm{C}(\%)$ & $7.8 \pm 1.8$ & $8.0 \pm 1.9$ & 0.429 \\
\hline $\mathrm{A} 1 \mathrm{C}(\mathrm{mmol} / \mathrm{mol})$ & $62 \pm 20$ & $64 \pm 21$ & 0.429 \\
\hline Total cholesterol $(\mathrm{mmol} / \mathrm{l})$ & $4.4 \pm 1.2$ & $4.3 \pm 1.1$ & 0.435 \\
\hline Triglyceride (mmol/l) & $1.3(1.0-2.0)$ & $1.4(1.1-1.8)$ & 0.374 \\
\hline HDL-C (mmol/l) & $1.3 \pm 0.3$ & $1.2 \pm 0.4$ & 0.867 \\
\hline LDL-C (mmol/l) & $2.6 \pm 0.9$ & $2.5 \pm 0.8$ & 0.298 \\
\hline hs-CRP (mg/dl) & $0.08(0.03-0.14)$ & $0.06(0.03-0.13)$ & 0.475 \\
\hline UAER (mg/gCr) & $12.9(7.3-38.0)$ & $14.8(8.3-105.8)$ & 0.216 \\
\hline eGFR $\left(\mathrm{ml} / \mathrm{min} / 1.73 \mathrm{~m}^{2}\right)$ & $96.5(85.7-106.1)$ & $93.5(71.4-100.8)$ & 0.016 \\
\hline $\mathrm{S} 1 \mathrm{P}(\mu \mathrm{mol} / \mathrm{l})$ & $9.5(7.2-12.0)$ & $8.1(6.4-11.2)$ & 0.024 \\
\hline Retinopathy, n (\%) & $42(19.5)$ & $20(27.8)$ & 0.141 \\
\hline Nephropathy, n (\%) & $25(11.6)$ & $17(23.6)$ & 0.013 \\
\hline Use of lipid-lowering agents, n (\%) & $106(49.3)$ & $34(47.2)$ & 0.760 \\
\hline Use of oral hypoglycemic agents, $\mathrm{n}(\%)$ & $131(60.9)$ & $50(69.4)$ & 0.195 \\
\hline Use of insulin, $\mathrm{n}(\%)$ & $25(11.6)$ & $12(16.7)$ & 0.269 \\
\hline Use of ACE inhibitors/ARBs, n (\%) & $80(37.2)$ & $34(47.2)$ & 0.133 \\
\hline Use of $\beta$-blockers, n (\%) & $18(8.4)$ & $10(13.9)$ & 0.172 \\
\hline Use of diuretics, n (\%) & $23(10.7)$ & $12(16.7)$ & 0.180 \\
\hline
\end{tabular}

Table 1. Characteristics of individuals with type 2 diabetes. Values are presented as the mean \pm standard deviation or median (interquartile range). Statistical testing by the chi-squared test, the Mann-Whitney U test or Student's t-test. A1C, glycated hemoglobin; ACE, angiotensin-converting enzyme; ARB, angiotensin II receptor blocker; CAN, cardiovascular autonomic neuropathy; eGFR, estimated glomerular filtration rate; HDL-C, high density lipoprotein cholesterol; hs-CRP, high-sensitivity C-reactive protein; LDL-C, low density lipoprotein cholesterol; S1P, Sphingosine 1-phosphate; UAER, urinary albumin excretion rate.

hypertension, A1C, high-sensitivity C-reactive protein (hs-CRP), diabetes duration, retinopathy and nephropathy (normal, $9.8 \mu \mathrm{mol} / 1$, 95\% CI 8.9-10.9; early, $9.0 \mu \mathrm{mol} / 1$, 95\% CI 8.0-10.0; definite, $7.1 \mu \mathrm{mol} / 1$, 95\% CI 6.2-8.1; $p$ for trend $=0.002$ ). In men, however, there was no significant difference in plasma S1P levels according to the degree of cardiovascular autonomic dysfunction.

Using logistic regression models, the association between plasma S1P levels and CAN was investigated in women and men with type $2 \mathrm{DM}$ (Table 3 ). In women, the relationship between plasma S1P levels and CAN was statistically significant (odds ratio [OR] per SD increase in the log-transformed value, $0.40 ; 95 \%$ CI, 0.23-0.70, $p=0.001$ ) after adjustment for age, BMI, HDL-C, LDL-C, triglycerides, hypertension, A1C, hs-CRP, diabetes duration, retinopathy, and nephropathy (model 3). In women, further adjustment for menopausal status did not alter the results (OR per SD increase in the log-transformed value, 0.39; 95\% CI, 0.22-0.69, $p=0.001$ ). However, no significant relationship was identified between plasma S1P levels and CAN in men. Alternatively, when hyperlipidemia and the use of insulin and OHAs were included as independent variables in the models and HDL-C, LDL-C, and triglycerides were excluded, plasma S1P levels were still associated with CAN in women (Supplementary Table 3).

\section{Discussion}

In the current study, we investigated the relationship between plasma S1P levels and CAN in individuals with type 2 DM. A significant interaction was identified between plasma S1P levels and sex with respect to CAN. When stratified by sex, the association between plasma S1P levels and CAN was observed only in women; plasma S1P levels were significantly and inversely associated with CAN in women after adjustment for well-known risk factors including A1C, DM duration, and hypertension. In addition, plasma S1P levels might be related to the severity of CAN in women with type $2 \mathrm{DM}$.

Recently, S1P has emerged as a bioactive signaling molecule, which influences a diverse range of cellular functions by acting as an extracellular signal or an intracellular second messenger ${ }^{5}$. S1P is much more abundant in blood than most tissues in which it is degraded via S1P lyase or S1P phosphatases ${ }^{14,15}$. It has been suggested 
$\mathbf{a}$
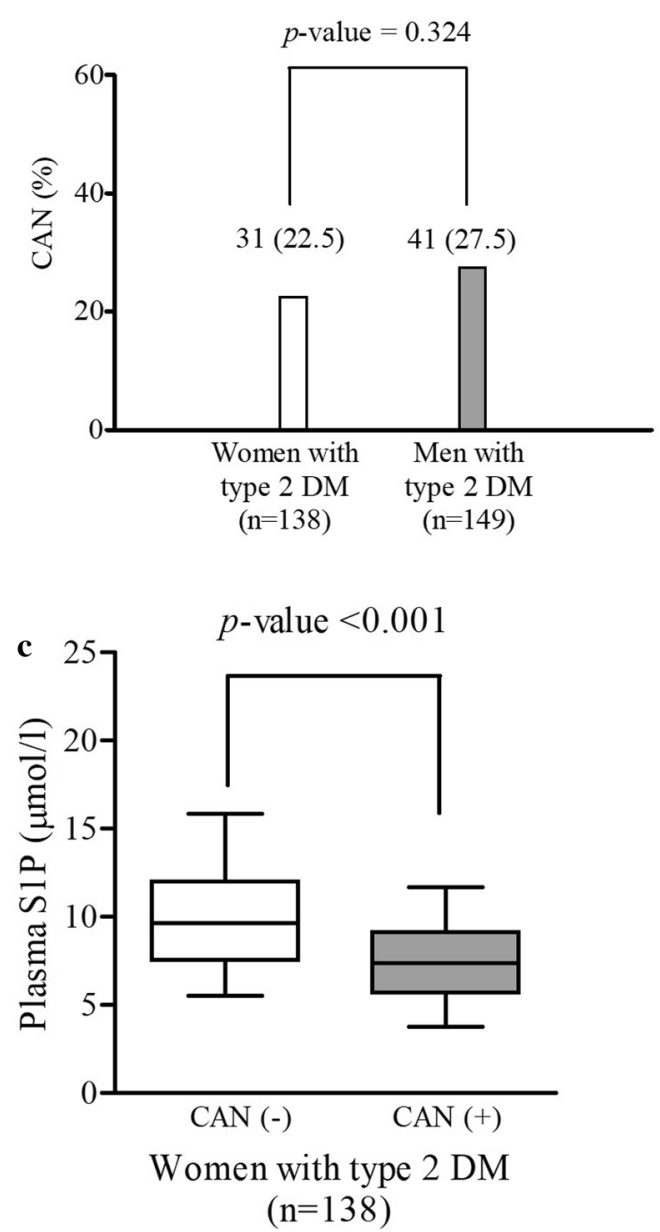

b $p$-value $=0.736$
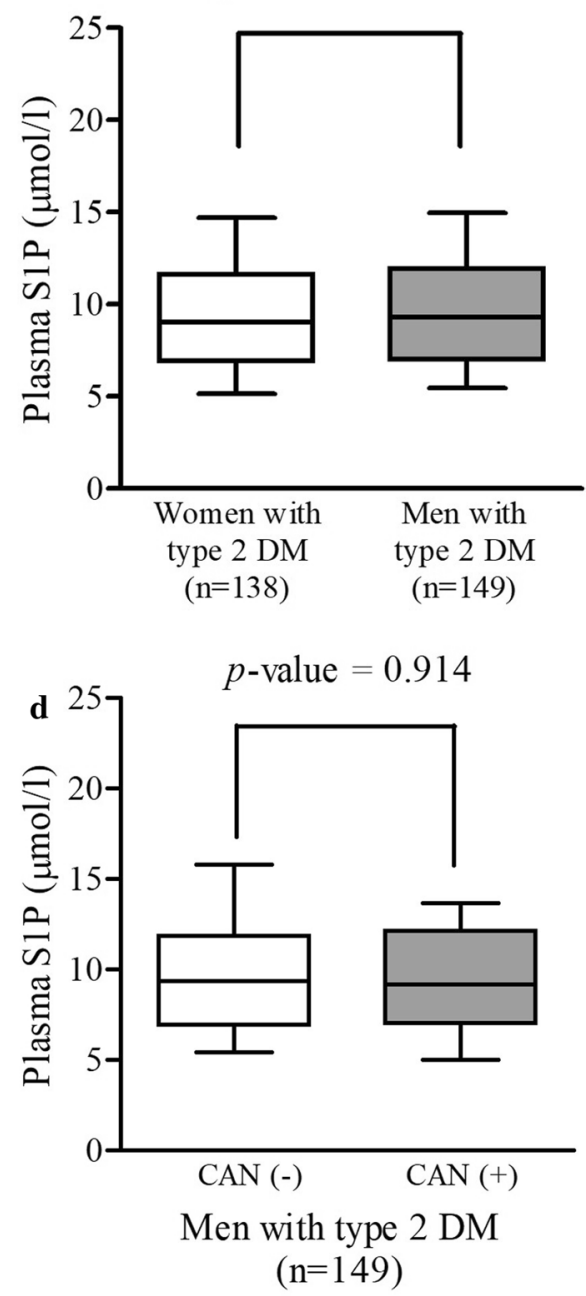

Figure 1. Characteristics of individuals with type 2 diabetes according to sex. (A) Prevalence of CAN according to sex. (B) Plasma S1P levels according to sex. (C) Plasma S1P levels in women according to CAN. (D) Plasma S1P levels in men according to CAN. Data are represented as frequencies (percentages). Statistical testing by the chi-squared test or the Mann-Whitney U test. Horizontal bars represent the 10th, 25th, 50th, 75th, and 90th percentile levels. CAN, cardiovascular autonomic neuropathy; S1P, sphingosine 1-phosphate.

\begin{tabular}{|l|l|l|l|l|l|}
\hline \multirow{2}{*}{ Plasma S1P $(\boldsymbol{\mu m o l} / \mathbf{l})$} & \multicolumn{2}{|l|}{ Degree of cardiovascular autonomic dysfunction } & \multirow{p}{*}{$\begin{array}{l}\boldsymbol{p} \text { for } \\
\text { trend }\end{array}$} \\
\cline { 2 - 6 } & Normal & Early & Definite & 0.003 \\
\hline \multirow{3}{*}{ Women } & Model 1 & $9.8(8.9-10.8)$ & $8.9(8.0-9.9)$ & $7.2(6.3-8.3)$ & 0.001 \\
\cline { 2 - 6 } & Model 2 & $9.9(8.9-10.9)$ & $8.9(8.0-9.9)$ & $7.1(6.1-8.1)$ & 0.002 \\
\cline { 2 - 6 } & Model 3 & $9.8(8.9-10.9)$ & $9.0(8.0-10.0)$ & $7.1(6.2-8.1)$ & 0.524 \\
\hline \multirow{3}{*}{ Men } & Model 1 & $9.4(8.4-10.6)$ & $8.7(7.7-9.7)$ & $9.4(8.2-10.8)$ & 0.165 \\
\cline { 2 - 6 } & Model 2 & $9.6(8.6-10.7)$ & $8.4(7.5-9.4)$ & $9.6(8.5-11.0)$ & 0.167 \\
\cline { 2 - 6 } & Model 3 & $9.6(8.6-10.9)$ & $8.4(7.5-9.5)$ & $9.8(8.5-11.3)$ & \\
\hline
\end{tabular}

Table 2. Comparison of plasma S1P levels according to the severity of cardiovascular autonomic dysfunction. S1P, sphingosine 1-phosphate. ${ }^{a}$ Values are presented as geometric mean (95\% confidence interval). Model 1: adjusted by age. Model 2: adjusted by model 1 plus body mass index, HDL-C, LDL-C, triglycerides ${ }^{\mathrm{a}}$, and hypertension. Model 3: adjusted by model 2 plus A1C, hs-CRPa , diabetes duration a , retinopathy, and nephropathy.

that the majority of S1P signaling occurs through binding to cell surface receptors ${ }^{16}$. S1P receptors are widely expressed in many tissues, particularly in the nervous, cardiovascular, and immune systems ${ }^{17}$. Therefore, S1P has been suggested as an easily accessible biomarker in clinical settings ${ }^{8}$. 


\begin{tabular}{|c|c|c|c|c|c|c|}
\hline \multirow{3}{*}{$\begin{array}{l}\text { Plasma S1Pa } \\
(\mu \mathrm{mol} / \mathrm{l})\end{array}$} & \multicolumn{6}{|c|}{ Cardiovascular autonomic neuropathy } \\
\hline & \multicolumn{3}{|c|}{ Women } & \multicolumn{3}{|l|}{ Men } \\
\hline & \begin{tabular}{|l|} 
OR \\
\end{tabular} & $95 \% \mathrm{CI}$ & $p$ value & OR & $95 \%$ CI & $p$ value \\
\hline Unadjusted & 0.43 & $0.27-0.69$ & $<0.001$ & 1.01 & $0.71-1.43$ & 0.971 \\
\hline Model 1 & 0.46 & $0.28-0.75$ & 0.002 & 1.10 & $0.76-1.60$ & 0.617 \\
\hline Model 2 & 0.43 & $0.25-0.72$ & 0.001 & 1.20 & $0.80-1.82$ & 0.379 \\
\hline Model 3 & 0.40 & $0.23-0.70$ & 0.001 & 1.25 & $0.78-1.99$ & 0.355 \\
\hline
\end{tabular}

Table 3. Logistic regression models of the association between S1P levels and cardiovascular autonomic neuropathy in individuals with type 2 diabetes according to sex. CI, confidence interval; OR, odds ratio; S1P, sphingosine 1-phosphate. ${ }^{a}$ Values were log-transformed prior to analysis. Model 1: adjusted by age. Model 2: adjusted by model 1 plus body mass index, HDL-C, LDL-C, triglycerides ${ }^{\mathrm{a}}$, and hypertension. Model 3: adjusted by model 2 plus A1C, hs-CRPa , diabetes duration ${ }^{\text {a }}$, retinopathy and nephropathy.

Previous investigations have reported that S1P may protect against the development of diabetes ${ }^{18-21}$. Previous clinical studies also showed that S1P levels were lower in patients with type $2 \mathrm{DM}$ compared with those without type $2 \mathrm{DM}^{22,23}$. In addition, accumulating evidence has demonstrated that S1P has crucial signaling functions in the nervous system ${ }^{6}$. Experimental studies have shown that S1P plays a pivotal role in neural development and may regulate neuronal differentiation, survival, excitability, arborization, and calcium signaling ${ }^{6,24,25}$. S1P was also found to be involved in neuronal repair ${ }^{6}$. Preclinical studies reported that administration of a S1P receptor agonist improved neuronal electrophysiologic function and reduced demyelination ${ }^{26,27}$. Szepanowski et al. ${ }^{28}$ also reported that treatment with a S1P receptor agonist enhanced axonal outgrowth after nerve injury, suggesting a beneficial role of S1P during the nerve regeneration process. In addition, reduced levels of S1P may be implicated in degenerative disorders in the nervous system ${ }^{8}$. Therefore, findings from previous studies have suggested that S1P may play a physiological neuroprotective role. However, the relationship between S1P and cardiovascular autonomic dysfunction remains unclear. To our knowledge, this is the first study to explore the relationship between plasma S1P levels and CAN in individuals with type $2 \mathrm{DM}$. Our results showed a significant and inverse relationship between plasma S1P levels and CAN in individuals, particularly women, with type 2 DM after adjustment for well-known risk factors for CAN. This suggests that S1P may be protective against cardiovascular autonomic dysfunction in type $2 \mathrm{DM}$ patients, although the causal nature of this association could not be confirmed in this study. Plasma S1P pool is suggested to be bound to HDL-C, LDL-C, and very low-density lipoprotein $s^{29}$. Several studies have reported that dyslipidemia is associated with $\mathrm{CAN}^{3,30}$. Thus, these lipoprotein abnormalities might contribute, in part, to the relationship between S1P and CAN in the current study. However, in the multivariable analysis, an association between S1P levels and CAN remained statistically significant, after adjustment for confounding factors including HDL-C, LDL-C, and triglycerides, indicating that these factors did not significantly influence the association between S1P and CAN.

Although the mechanisms by which S1P is linked to CAN remain unclear, plausible explanations exist. S1P is related to oxidative stress ${ }^{31}$ and endothelial function ${ }^{8}$, and S1P is known to decrease oxidative stress in neuronal cells ${ }^{31}$. Furthermore, $\mathrm{S} 1 \mathrm{P}$ is thought to enhance endothelial barrier function, and to be involved in the maintenance of vascular integrity and the repair of vascular injury ${ }^{8}$. Chronic hyperglycemia results in oxidative stress, and increased oxidative stress induces neuronal damage ${ }^{1}$. Endothelial damage also impairs neuronal blood flow ${ }^{32}$. These pathways are considered to be critical contributors to the pathogenesis of autonomic neuropathy ${ }^{32,33}$. In addition, S1P is involved in inflammation and immune reactions ${ }^{34}$. Therefore, even though the causal relationships could not be established in this study, these findings indicate that S1P plays a protective role in the pathogenesis of CAN.

In the present study, the association between S1P levels and CAN in individuals with type 2 DM showed a sex difference. Although the reason for this sex difference remains unclear, sex hormones may underlie this sexspecific association between S1P levels and CAN. Several studies have suggested that estrogen might modulate the autonomic nervous system ${ }^{35}$. Estrogen treatment has previously been reported to decrease sympathetic drive and ameliorate cardiac autonomic control ${ }^{36}$. Furthermore, in vitro studies, estrogen has been shown to enhance the synthesis and export of S1P from cancer cell lines ${ }^{37}$. In the study of 108 healthy participants, Guo et al. ${ }^{38}$ reported that plasma S1P levels were higher in women than in men. In our study, however, there was no significant difference in the prevalence of CAN between women and men, which correlates with previous studies ${ }^{39,40}$. Unlike the findings reported by Guo et al. ${ }^{38}$, in this study, plasma S1P levels did not significantly differ between women and men with type $2 \mathrm{DM}$, which may be a result of differences in study populations. Moreover, in our study, menopausal status did not significantly affect the relationship between plasma S1P levels and CAN in women. Thus, it suggests that additional mechanisms might exist. HDL may be in part implicated in a sex-specific association between S1P levels and CAN. Previous studies have suggested that higher levels of HDL particles are observed in women compared with men ${ }^{41,42}$. HDL function is partly dependent on S1P. HDL-associated S1P may contribute to the anti-oxidative and anti-inflammatory functions of HDL, which relate to protective roles against vascular damage $^{43,44}$. Additionally, a sex difference might be assumed to be due to adverse impacts of androgen in men, although it is currently unproven ${ }^{45}$. Further studies are therefore required to explore the mechanism underlying the sex difference in association between S1P levels and CAN.

We note that ceramide promotes apoptosis, which is linked to an opposing action to $\mathrm{S}_{\mathrm{P}} \mathrm{P}^{5}$. In an experimental study, apoptosis of mouse Schwann cells was enhanced by incubation with palmitate, and these effects were 
suppressed by inhibition of serine-palmitoyl transferase, the rate-limiting step of ceramide biosynthesis, suggesting a potential role of ceramide in nerve damage ${ }^{46}$. In contrast, Dohrn et al ${ }^{47}$ found no significant differences in plasma levels of the ceramide precursor sphinganine, including C16-, C17-, C18-, and C20-sphinganine, between type $2 \mathrm{DM}$ patients with distal sensorimotor polyneuropathy and healthy controls. Therefore, future studies to investigate the role of ceramide in CAN would provide additional information to better understand pathogenic mechanisms of S1P in cardiovascular autonomic dysfunction.

There are some limitations in our study. First, because of the cross-sectional nature of the study, the causal relationships could not be ascertained. Second, although mass spectrometry-based assay is suggested as the gold standard technique for sphingolipid analysis ${ }^{48}$, the method using commercial ELISA kits has been increasingly used to measure S1P level ${ }^{10,49,50}$. In addition, this method used in our study was previously validated by liquid chromatography-tandem mass spectrometry ${ }^{51}$. Finally, although each of HR variability tests has good sensitivity and specificity of $\sim 90 \%{ }^{1}$ and abnormal HR variability in one test indicates early autonomic neuropathy ${ }^{1,4}$, these tests might not be able to detect abnormalities in early subclinical CAN. Thus, early involvement of cardiovascular autonomic dysfunction might be underestimated in this study. However, because this would bias toward the null, we believe that our findings would not be significantly affected. Despite these limitations, our data might give an important information with regard to relationships between plasma S1P levels and CAN in type 2 DM patients.

In conclusion, we identified an inverse relationship between plasma S1P levels and CAN only in women with type $2 \mathrm{DM}$. Further studies are necessary to determine the mechanism underlying the sex difference in the association between S1P levels and CAN.

Received: 27 January 2020; Accepted: 15 July 2020

Published online: 29 July 2020

\section{References}

1. Vinik, A. I., Maser, R. E., Mitchell, B. D. \& Freeman, R. Diabetic autonomic neuropathy. Diabetes Care 26, 1553-1579. https://doi. org/10.2337/diacare.26.5.1553 (2003).

2. Vinik, A. I., Erbas, T. \& Casellini, C. M. Diabetic cardiac autonomic neuropathy, inflammation and cardiovascular disease. J. Diabetes Investig. 4, 4-18. https://doi.org/10.1111/jdi.12042 (2013).

3. Pop-Busui, R. et al. Diabetic neuropathy: a position statement by the American Diabetes Association. Diabetes Care 40, 136-154. https://doi.org/10.2337/dc16-2042 (2017).

4. Pop-Busui, R. Cardiac autonomic neuropathy in diabetes: a clinical perspective. Diabetes Care 33, 434-441. https://doi.org/10.2337/ dc09-1294 (2010).

5. Fyrst, H. \& Saba, J. D. An update on sphingosine-1-phosphate and other sphingolipid mediators. Nat. Chem. Biol. 6, $489-497$. https://doi.org/10.1038/nchembio.392 (2010).

6. Soliven, B., Miron, V. \& Chun, J. The neurobiology of sphingosine 1-phosphate signaling and sphingosine 1-phosphate receptor modulators. Neurology 76, S9-14. https://doi.org/10.1212/WNL.0b013e31820d9507 (2011).

7. Szepanowski, F. \& Kieseier, B. C. Targeting lysophospholipid signaling as a therapeutic approach towards improved peripheral nerve regeneration. Neural Regen. Res. 11, 1754-1755. https://doi.org/10.4103/1673-5374.194720 (2016).

8. Proia, R. L. \& Hla, T. Emerging biology of sphingosine-1-phosphate: its role in pathogenesis and therapy. J. Clin. Invest. 125, 1379-1387. https://doi.org/10.1172/JCI76369 (2015).

9. Expert Committee on the Diagnosis and Classification of Diabetes Mellitus. Report of the expert committee on the diagnosis and classification of diabetes mellitus. Diabetes Care 26(Suppl 1), S5-S20. https://doi.org/10.2337/diacare.26.2007.s5 (2003).

10. Lai, W. Q. et al. Anti-inflammatory effects of sphingosine kinase modulation in inflammatory arthritis. J. Immunol. 181, 8010-8017. https://doi.org/10.4049/jimmunol.181.11.8010 (2008).

11. Levey, A. S. et al. A new equation to estimate glomerular filtration rate. Ann. Intern. Med. 150, 604-612. https://doi. org/10.7326/0003-4819-150-9-200905050-00006 (2009).

12. Ewing, D. J., Martyn, C. N., Young, R. J. \& Clarke, B. F. The value of cardiovascular autonomic function tests: 10 years experience in diabetes. Diabetes Care 8, 491-498. https://doi.org/10.2337/diacare.8.5.491 (1985).

13. Tesfaye, S. et al. Diabetic neuropathies: update on definitions, diagnostic criteria, estimation of severity, and treatments. Diabetes Care 33, 2285-2293. https://doi.org/10.2337/dc10-1303 (2010).

14. Pappu, R. et al. Promotion of lymphocyte egress into blood and lymph by distinct sources of sphingosine-1-phosphate. Science 316, 295-298. https://doi.org/10.1126/science.1139221 (2007).

15. Zhao, Y. et al. Intracellular generation of sphingosine 1-phosphate in human lung endothelial cells: role of lipid phosphate phosphatase-1 and sphingosine kinase 1. J. Biol. Chem. 282, 14165-14177. https://doi.org/10.1074/jbc.M701279200 (2007).

16. O'Sullivan, S. \& Dev, K. K. Sphingosine-1-phosphate receptor therapies: advances in clinical trials for CNS-related diseases. Neuropharmacology 113,597-607. https://doi.org/10.1016/j.neuropharm.2016.11.006 (2017).

17. Chae, S. S., Proia, R. L. \& Hla, T. Constitutive expression of the S1P1 receptor in adult tissues. Prostaglandins Other Lipid Mediat. 73, 141-150 (2004).

18. Rapizzi, E. et al. Sphingosine 1-phosphate increases glucose uptake through trans-activation of insulin receptor. Cell Mol. Life Sci. 66, 3207-3218. https://doi.org/10.1007/s00018-009-0106-3 (2009).

19. Moon, M. H. et al. Antiobesity activity of a sphingosine 1-phosphate analogue FTY720 observed in adipocytes and obese mouse model. Exp. Mol. Med. 44, 603-614. https://doi.org/10.3858/emm.2012.44.10.069 (2012).

20. Zhao, Z., Choi, J., Zhao, C. \& Ma, Z. A. FTY720 normalizes hyperglycemia by stimulating beta-cell in vivo regeneration in db/db mice through regulation of cyclin D3 and p57(KIP2). J. Biol. Chem. 287, 5562-5573. https://doi.org/10.1074/jbc.M111.305359 (2012).

21. Qi, Y. et al. Loss of sphingosine kinase 1 predisposes to the onset of diabetes via promoting pancreatic beta-cell death in dietinduced obese mice. FASEB J. 27, 4294-4304. https://doi.org/10.1096/fj.13-230052 (2013).

22. Sui, J. et al. Sphingolipid metabolism in type 2 diabetes and associated cardiovascular complications. Exp. Ther. Med. 18, 3603-3614. https://doi.org/10.3892/etm.2019.7981 (2019).

23. Vaisar, T. et al. Type 2 diabetes is associated with loss of HDL endothelium protective functions. PLoS ONE 13, e0192616. https:// doi.org/10.1371/journal.pone.0192616 (2018).

24. Herr, D. R. \& Chun, J. Effects of LPA and S1P on the nervous system and implications for their involvement in disease. Curr. Drug Targets 8, 155-167 (2007).

25. Kanno, T. et al. Regulation of synaptic strength by sphingosine 1-phosphate in the hippocampus. Neuroscience 171, 973-980. https ://doi.org/10.1016/j.neuroscience.2010.10.021 (2010). 
26. Foster, C. A. et al. FTY720 rescue therapy in the dark agouti rat model of experimental autoimmune encephalomyelitis: expression of central nervous system genes and reversal of blood-brain-barrier damage. Brain Pathol. 19, 254-266. https://doi.org/10.111 1/j.1750-3639.2008.00182.x (2009).

27. Balatoni, B. et al. FTY720 sustains and restores neuronal function in the DA rat model of MOG-induced experimental autoimmune encephalomyelitis. Brain Res. Bull. 74, 307-316. https://doi.org/10.1016/j.brainresbull.2007.06.023 (2007).

28. Szepanowski, F. et al. Fingolimod promotes peripheral nerve regeneration via modulation of lysophospholipid signaling. J. Neuroinflamm. 13, 143. https://doi.org/10.1186/s12974-016-0612-9 (2016).

29. Argraves, K. M. \& Argraves, W. S. HDL serves as a S1P signaling platform mediating a multitude of cardiovascular effects. J. Lipid Res. 48, 2325-2333. https://doi.org/10.1194/jlr.R700011-JLR200 (2007).

30. Voulgari, C. et al. The association between cardiac autonomic neuropathy with metabolic and other factors in subjects with type 1 and type 2 diabetes. J. Diabetes Complicat. 25, 159-167. https://doi.org/10.1016/j.jdiacomp.2010.06.001 (2011).

31. Pyszko, J. \& Strosznajder, J. B. Sphingosine kinase 1 and sphingosine-1-phosphate in oxidative stress evoked by 1-methyl-4-phenylpyridinium (MPP+) in human dopaminergic neuronal cells. Mol. Neurobiol. 50, 38-48. https://doi.org/10.1007/s12035-0138622-4 (2014).

32. Kuehl, M. \& Stevens, M. J. Cardiovascular autonomic neuropathies as complications of diabetes mellitus. Nat. Rev. Endocrinol. 8, 405-416. https://doi.org/10.1038/nrendo.2012.21 (2012).

33. Pop-Busui, R., Sima, A. \& Stevens, M. Diabetic neuropathy and oxidative stress. Diabetes Metab. Res. Rev. 22, 257-273. https:// doi.org/10.1002/dmrr.625 (2006)

34. Spiegel, S. \& Milstien, S. The outs and the ins of sphingosine-1-phosphate in immunity. Nat. Rev. Immunol. 11, 403-415. https:// doi.org/10.1038/nri2974 (2011).

35. Dart, A. M., Du, X. J. \& Kingwell, B. A. Gender, sex hormones and autonomic nervous control of the cardiovascular system. Cardiovasc. Res. 53, 678-687. https://doi.org/10.1016/s0008-6363(01)00508-9 (2002).

36. Gokce, M. et al. Long term effects of hormone replacement therapy on heart rate variability, QT interval, QT dispersion and frequencies of arrhythmia. Int. J. Cardiol. 99, 373-379. https://doi.org/10.1016/j.ijcard.2003.03.030 (2005).

37. Takabe, K. et al. Estradiol induces export of sphingosine 1-phosphate from breast cancer cells via ABCC1 and ABCG2. J. Biol. Chem. 285, 10477-10486. https://doi.org/10.1074/jbc.M109.064162 (2010).

38. Guo, S. et al. Higher level of plasma bioactive molecule sphingosine 1-phosphate in women is associated with estrogen. Biochim. Biophys. Acta 836-846, 2014. https://doi.org/10.1016/j.bbalip.2014.02.005 (1841).

39. Moran, A. et al. Cardiovascular autonomic neuropathy is associated with microalbuminuria in older patients with type 2 diabetes. Diabetes Care 27, 972-977. https://doi.org/10.2337/diacare.27.4.972 (2004).

40. Kempler, P. et al. Autonomic neuropathy is associated with increased cardiovascular risk factors: the EURODIAB IDDM complications study. Diabetes Med. 19, 900-909. https://doi.org/10.1046/j.1464-5491.2002.00821.x (2002).

41. Jeyarajah, E. J., Cromwell, W. C. \& Otvos, J. D. Lipoprotein particle analysis by nuclear magnetic resonance spectroscopy. Clin. Lab. Med. 26, 847-870. https://doi.org/10.1016/j.cll.2006.07.006 (2006).

42. Woudberg, N. J. et al. Pharmacological intervention to modulate HDL: what do we target?. Front. Pharmacol. 8, 989. https://doi. org/10.3389/fphar.2017.00989 (2017).

43. Tolle, M. et al. HDL-associated lysosphingolipids inhibit $\mathrm{NAD}(\mathrm{P}) \mathrm{H}$ oxidase-dependent monocyte chemoattractant protein-1 production. Arterioscler. Thromb. Vasc. Biol. 28, 1542-1548. https://doi.org/10.1161/ATVBAHA.107.161042 (2008).

44. Brulhart-Meynet, M. C. et al. Improving reconstituted HDL composition for efficient post-ischemic reduction of ischemia reperfusion injury. PLoS ONE 10, e0119664. https://doi.org/10.1371/journal.pone.0119664 (2015).

45. Rossouw, J. E. Hormones, genetic factors, and gender differences in cardiovascular disease. Cardiovasc. Res. 53, 550-557. https:// doi.org/10.1016/s0008-6363(01)00478-3 (2002).

46. Suzuki, J. et al. Palmitate induces apoptosis in Schwann cells via both ceramide-dependent and independent pathways. Neuroscience 176, 188-198. https://doi.org/10.1016/j.neuroscience.2010.11.035 (2011).

47. Dohrn, M. F. et al. Elevation of plasma 1-deoxy-sphingolipids in type 2 diabetes mellitus: a susceptibility to neuropathy?. Eur. J. Neurol. 22, 806-814. https://doi.org/10.1111/ene.12663 (2015).

48. Blachnio-Zabielska, A. U., Persson, X. M., Koutsari, C., Zabielski, P. \& Jensen, M. D. A liquid chromatography/tandem mass spectrometry method for measuring the in vivo incorporation of plasma free fatty acids into intramyocellular ceramides in humans. Rapid Commun. Mass Spectrom. 26, 1134-1140. https://doi.org/10.1002/rcm.6216 (2012).

49. Gomaraschi, M. et al. Inflammation impairs eNOS activation by HDL in patients with acute coronary syndrome. Cardiovasc. Res. 100, 36-43. https://doi.org/10.1093/cvr/cvt169 (2013).

50. Cao, C. et al. The efficacy of activated protein C in murine endotoxemia is dependent on integrin CD11b. J. Clin. Invest. 120, 1971-1980. https://doi.org/10.1172/JCI40380 (2010).

51. Kirby, R. J. et al. Dynamic regulation of sphingosine-1-phosphate homeostasis during development of mouse metanephric kidney. Am. J. Physiol. Renal Physiol. 296, F634-641. https://doi.org/10.1152/ajprenal.90232.2008 (2009).

\section{Acknowledgements}

This study was supported by the National Research Foundation of Korea (NRF) grant funded by the Korea government (MSIT) (No. 2018R1C1B5045647) and Chonnam National University (Grant Number: 2018-3551).

\section{Author contributions}

M.Y.C. and J.O.C. designed the study, drafted the manuscript, and approved its final version. S.Y.P. and J.O.C. contributed to the statistical analyses and interpretation of data. J.O.C., D.H.C., D.J.C., and M.Y.C. acquired data. All authors reviewed and approved the final manuscript.

\section{Competing interests}

The authors declare no competing interests.

\section{Additional information}

Supplementary information is available for this paper at https://doi.org/10.1038/s41598-020-69566-y.

Correspondence and requests for materials should be addressed to J.O.C.

Reprints and permissions information is available at www.nature.com/reprints.

Publisher's note Springer Nature remains neutral with regard to jurisdictional claims in published maps and institutional affiliations. 
(c) (i) Open Access This article is licensed under a Creative Commons Attribution 4.0 International cc) License, which permits use, sharing, adaptation, distribution and reproduction in any medium or format, as long as you give appropriate credit to the original author(s) and the source, provide a link to the Creative Commons license, and indicate if changes were made. The images or other third party material in this article are included in the article's Creative Commons license, unless indicated otherwise in a credit line to the material. If material is not included in the article's Creative Commons license and your intended use is not permitted by statutory regulation or exceeds the permitted use, you will need to obtain permission directly from the copyright holder. To view a copy of this license, visit http://creativecommons.org/licenses/by/4.0/.

(C) The Author(s) 2020 